\title{
Affiliate network members as force amplifiers of genomic medicine research
}

\author{
Geoffrey S Ginsburg*,1 (iD), Ebony Madden² \& Philip E Empey ${ }^{3}$ \\ ${ }^{1}$ Duke Center for Applied Genomics \& Precision Medicine, Duke University, Durham, NC 27708, USA \\ ${ }^{2}$ Division of Genomic Medicine, National Human Genome Research Institute (NHGRI), Bethesda, MD 20892, USA \\ ${ }^{3}$ University of Pittsburgh Medical Center (UPMC) Institute of Precision Medicine, University of Pittsburgh, Pittsburgh, PA 15213 USA \\ *Author for correspondence: geoffrey.ginsburg@duke.edu
}

"we recognize Affiliate Members as a force multiplier for network goals and productivity. We believe that participation models that incentivize broad involvement are needed to maximize the impact of these research networks."

First draft submitted: 6 July 2019; Accepted for publication: 6 August 2019; Published online: 11 November 2019

Keywords: affiliate $\bullet$ genomic $\bullet$ methodology $\bullet$ network $\bullet$ pharmacogenomic

The Implementing GeNomics In pracTicE (IGNITE) Network is an NIH-funded network dedicated to advancing the implementation of genomics in healthcare [1]. The network is comprised of six research sites, a coordinating center, a steering committee and working groups. Starting in 2013, the IGNITE network initiated six distinct genomic medicine projects aimed at implementation of validated genome-based technologies into clinical workflows and the electronic medical record as well as the development of clinical decision support for providers. A goal of the IGNITE Network is to disseminate the methods and best practices from these projects in order to advance the implementation of genomics in healthcare. During the 5 years since the initiation of the network, groups from across the country, who were not funded by NIH, expressed interest in joining the network for the purposes of both learning about genomic medicine implementation that they could use locally and for sharing their experiences in doing so with network members. Thus, Network Affiliate Members have become a key asset to the network. Network communications, and arguably their strength, is proportional to its number of members (known as Metcalf's Law [2]). Here we present the IGNITE experience of network enhancements that have been achieved by the incorporation of affiliate members and their organizations.

The IGNITE Network made the strategic decision to allow both academic and industry Affiliate Members to the network as the network's goals to implement genomic medicine in diverse care settings and the goals of industry to effectively penetrate markets to see products are aligned in many respects. Both require stakeholder engagement, an understanding of workflows and challenges to bringing new technologies into them, and develop an evidence base that supports adoptions. Thus, there are latent synergies between these two communities that can be very advantageous in the development of broad implementation programs. Also, as one of the the goals of the IGNITE Network is to expand genomic medicine outside of the academic settings, Affiliate Members increase the expansion outside of the adopter sites that were funded by the grants.

IGNITE has 18 Affiliate Members from both academia and industry and designated investigators from each (Supplementary Figure 1 courtesy of the IGNITE Administrative Coordinating Center at Duke University and the National Human Genome Research Institute). Fifteen Affiliate Members are from academic settings, and three are from industry. Recruiting Affiliate Members and effective onboarding was an early goal of the network. Joining involved signing a memorandum of understanding to set expectations regarding participation (available by request from authors). Specifically, to be involved in network meetings and working groups, to share implementation data (within data use agreements when needed), and to adhere to authorship and publication guidelines. As a result of the affiliate participation, the network has been enriched in geographic diversity of the sites, diversity of the healthcare delivery systems and their demographics and diversity of experiences, particularly in the community setting. Affiliate members are given an opportunity to present their local programs and implementation challenges

Future Medicine 
and strategy at network meetings. Thus, the network's NIH-funded core investigator community has benefited from the significant expansion of the range of genomic medicine experiences and implementation strategies that the affiliates bring to the conversations and the overall knowledge of the network.

A recent survey of Affiliate Members indicated that $70 \%$ had attended in person meetings, $100 \%$ were on one or more working groups, $60 \%$ had contributed to one or more publications and had contributed data and $100 \%$ of the IGNITE working groups had affiliate involvement (unpublished data from the IGNITE Network). A noteworthy example is the contribution to the Pharmacogenetics (PGx) working group from Affiliate Members. Seventeen IGNITE Affiliate Member organizations participated in this working group. Affiliates have been co-authors on three cross network papers published to date [3-5] with eight affiliates contributing data to these papers and one led by an Affiliate Member [5]. Importantly, affiliate-contributed data added a significant number of subjects to the analysis increasing power, the diversity of settings and thus the significance of the findings. In fact, in one paper, the affiliate dataset contribution surpassed the IGNITE-funded site contributions [4]. In another, 12 institutions contributed to compare and contrast optimal strategies for PGx implementation in the cardiac catheterization laboratory [5].

One of the goals of the network is to disseminate best practices and knowledge the network acquires in how to implement genomic medicine. A platform that enables dissemination is the network's SPARK toolbox [6]. Five network affiliates have contributed to the toolbox and also served to test the tools and provide feedback for their optimization and utility. Real world users of the tools, who are invested in the network, have been a trusted test bed for the network's dissemination strategy. Moreover, the affiliates have also become vehicles for communicating the network's findings to their provider communities locally and also to a broader audience through conferences and seminars.

IGNITE involvement also provided direct benefits to Affiliate Members who sought to start or expand genomic implementation programs (Supplementary Table 1). Many of the contributions to the network described above simultaneously provided value back to Affiliate Members and their institutions. Through in-person meetings, conference calls and workgroup involvement, Affiliate Members developed close working relationships with peers facing common implementation barriers and learned from the successes of these early adopters. Real world implementation solutions to deploying genomic testing, electronic health record configuration to support genomics, feasible clinical workflows and keys to success were routinely discussed. The sharing of outcome data increased the power of analyses earlier than achievable independently and co-authored publications increased the profile of local efforts. For example, in the IGNITE PGx working group's pragmatic clinical trial of CYP2C19 genotyping following cardiac catheterization, differences in outcomes with genotype-guided antiplatelet therapy were reported when data on 1815 patients were combined from seven sites with similar implementations [4].

When surveyed about their experiences, one affiliate responded that "being an IGNITE affiliate has been helpful, as we are able to hear implementation experiences and use implementation tools created by others who have gone before us." Another noted "we are at the initial stages of (genomics) implementation and the resources and collaborations provided by the network have been instrumental in our preparation" IGNITE Network [unpublished data]. Indeed, the barriers to genomic medicine implementation are well recognized and the motivation to learn from others was cited as a key driver to participation. Importantly, this affiliate involvement meets the network's goal advance genomic medicine in patient care and the results are quantifiable. Of the academic Affiliate Members, $83 \%$ had existing implementation projects and 58\% launched new or additional genomics implementation projects after joining the network.

The high affiliate engagement is unique among large research networks and should be noted as a key success of IGNITE. Large research networks have not typically engaged many sites beyond the core, funded institutions. For example, the Electronic Medical Records and Genomics (eMERGE) [7] and Clinical Sequencing EvidenceGenerating Research (CSER) [8] networks have nine and seven core sites with only six and no Affiliate Members, respectively. Whether participation in IGNITE was because of its focus (genomic implementation), a particular domain, the investigators involved, early dissemination network successes, active methods of engagement or other reasons, is unknown.

Overall, as we close the final year of the initial IGNITE network, we recognize Affiliate Members as a force multiplier for network goals and productivity. We believe that participation models that incentivize broad involvement are needed to maximize the impact of these research networks. Long term, sustainability is of concern and several Affiliate Members suggested developing funding mechanisms to further incentivize participation. This was noted to be important specifically for resource intensive activities such as data gathering and sharing was requested. 
As the IGNITE network transitions to its next phase, 100\% of Affiliate Members surveyed expressed eagerness to maintain involvement in the network. The IGNITE experience is an exemplar of success in leveraging affiliates to extend research network goals.

\section{Supplementary data}

To view the supplementary data that accompany this paper please visit the journal website at: www.futuremedicine.com/doi/suppl/10.2217/pme-2019-0077

\section{Financial \& competing interests disclosure}

This paper was supported by a grant from the National Human Genome Research Institute (NIH-HG007282-04S1). The authors have no other relevant affiliations or financial involvement with any organization or entity with a financial interest in or financial conflict with the subject matter or materials discussed in the manuscript apart from those disclosed.

No writing assistance was utilized in the production of this manuscript.

\section{References}

1. Weitzel KW, Alexander M, Bernhardt BA et al. The IGNITE network: a model for genomic medicine implementation and research. BMC Med. Genomics 9(1), 1 (2016).

2. Metcalfe's Law (2019). https://en.wikipedia.org/wiki/Metcalfe\%27s_law

3. Cavallari LH, Beitelshees AL, Blake KV et al. The IGNITE pharmacogenetics working group: an opportunity for building evidence with pharmacogenetic implementation in a real-world setting. Clin. Transl. Sci. 10, 143-146 (2017).

4. Cavallari LH, Lee CR, Beitelshees AL et al. Multisite investigation of outcomes with implementation of CYP2C19 genotype-guided antiplatelet therapy after percutaneous coronary intervention. JACC Cardiovasc. Interv. 11, 181-191 (2018).

5. Empey PE, Stevenson JM, Tuteja S et al. Multisite investigation of strategies for the implementation of CYP2C19 genotype-guided antiplatelet therapy. Clin. Pharmacol. Ther. 104, 664-674 (2018).

6. IGNITE (2019). https://ignite-genomics.org/spark-toolbox/

7. Emerge Network (2019). https://emerge.mc.vanderbilt.edu/

8. Clinical Sequencing Evidence-Generating Research (2019) www.genome.gov/27546194/clinical-sequencing-evidencegenerating-research-cser2/ 